\title{
Deep UV Formation of Long-Term Stable Optical Bragg Gratings in Epoxy Waveguides and Their Biomedical Sensing Potentials
}

\author{
Steffen Hessler $^{1, *(\mathbb{D})}$, Marieke Rüth ${ }^{2} \mathbb{D}$, Horst-Dieter Lemke ${ }^{2}$, Bernhard Schmauss $^{3} \mathbb{D}_{\text {and Ralf Hellmann }}{ }^{1}$ \\ 1 Applied Laser and Photonics Group, Faculty of Engineering, University of Applied Sciences Aschaffenburg, \\ Würzburger Straße 45, 63743 Aschaffenburg, Germany; ralf.hellmann@th-ab.de \\ 2 eXcorLab GmbH, Industrie Center Obernburg, 63784 Obernburg am Main, Germany; \\ marieke.rueth@excorlab.de (M.R.); horstdieter.lemke@excorlab.de (H.-D.L.) \\ 3 Institute of Microwaves and Photonics, University of Erlangen-Nuremberg, Cauerstraße 9, \\ 91054 Erlangen, Germany; bernhard.schmauss@fau.de \\ * Correspondence: steffen.hessler@th-ab.de
}

\section{check for} updates

Citation: Hessler, S.; Rüth, M.; Lemke, H.-D.; Schmauss, B.; Hellmann, R. Deep UV Formation of Long-Term Stable Optical Bragg Gratings in Epoxy Waveguides and Their Biomedical Sensing Potentials. Sensors 2021, 21, 3868. https:// doi.org/10.3390/s21113868

Academic Editors: Aitor Urrutia, Pablo Zubiate, Nerea De Acha Morrás and Diego Lopez-Torres

Received: 17 May 2021

Accepted: 31 May 2021

Published: 3 June 2021

Publisher's Note: MDPI stays neutral with regard to jurisdictional claims in published maps and institutional affiliations.

Copyright: (c) 2021 by the authors. Licensee MDPI, Basel, Switzerland. This article is an open access article distributed under the terms and conditions of the Creative Commons Attribution (CC BY) license (https:// creativecommons.org/licenses/by/ $4.0 /)$.

\begin{abstract}
In this article, we summarize our investigations on optimized $248 \mathrm{~nm}$ deep ultraviolet (UV) fabrication of highly stable epoxy polymer Bragg grating sensors and their application for biomedical purposes. Employing m-line spectroscopy, deep UV photosensitivity of cross-linked EpoCore thin films in terms of responding refractive index change is determined to a maximum of $\Delta \mathrm{n}=+(1.8 \pm 0.2) \times 10^{-3}$. All-polymer waveguide Bragg gratings are fabricated by direct laser irradiation of lithographic EpoCore strip waveguides on compatible Topas 6017 substrates through standard $+1 /$-1-order phase masks. According near-field simulations of realistic non-ideal phase masks provide insight into UV dose-dependent characteristics of the Bragg grating formation. By means of online monitoring, arising Bragg reflections during grating inscription via beforehand fiber-coupled waveguide samples, an optimum laser parameter set for well-detectable sensor reflection peaks in respect of peak strength, full width at half maximum and grating attenuation are derived. Promising blood analysis applications of optimized epoxy-based Bragg grating sensors are demonstrated in terms of bulk refractive index sensing of whole blood and selective surface refractive index sensing of human serum albumin.
\end{abstract}

Keywords: optical sensor; Bragg grating; EpoCore; excimer laser; refractive index sensing; blood analysis; human serum albumin; rapid test

\section{Introduction}

Over the past two decades, Bragg grating technology has emerged as an attractive optical sensor tool enabling versatile multi-physical parameter monitoring [1-4]. On the one hand, optical glass fiber Bragg grating (FBG) based sensors are frequently applied in the fields of mechanical structural health monitoring and temperature sensing, confirming commercial application maturity [5-8]. In addition, Bragg grating sensors created in polymer optical fibers (POFs) have widely been demonstrated, e.g., for popular biomedical healthcare applications $[9,10]$. On the other hand, a multitude of highly sensitive planar Bragg grating (PBG) based sensing concepts, typically in the form of silica and silicon integrated optical waveguide chips, have been investigated for most diverse application scenarios. By utilizing silicon nano-waveguide Bragg grating structures, on-chip and small footprint temperature sensors have been developed [11]. Directly laser-written PBGs realized in doped fused silica layers were efficiently employed as refractive index sensors for fermentation monitoring, concentration measurements of binary mixtures, and for biomedical substance detection using antibody surface layers [12-15]. Additionally, significant chemosensory potential for trace gas detection of harmful organic compounds [16] and 
ozone depleting trichlorofluoromethane was demonstrated using silica-based PBG sensors which were surface functionalized with cyclodextrin capture coatings [17,18].

Since a material substitution by optical polymers, instead, is in general accompanied by notable advantages such as biocompatibility as well as less intricate and costly fabrication procedures besides broadly tunable optical, thermal, and mechanical properties [19,20], polymer-based PBG designs suggest further appealing and particularly inexpensive sensor solutions beyond the material limits of silica and silicon. For instance, two-dimensional mechanical strain sensing as well as humidity insensitive shape sensing were achieved with polymer Bragg gratings in bulk polymethylmethacrylate (PMMA) and cyclic olefin copolymer (COC) substrates [21,22]. Moreover, the cross-linked optical epoxy polymer negative photoresist EpoCore (micro resist technology) turned out to offer exceptional material properties such as high mechanical and extreme chemical robustness [23] compared with COC and PMMA. Owing to EpoCore's ultraviolet (UV) photosensitivity, precise waveguide geometries can be defined by standard $365 \mathrm{~nm}$ photolithography processes. The cured epoxy resist provides polymer-typical optical transparency windows around the primary wavelengths $850 \mathrm{~nm}, 1310 \mathrm{~nm}$, and $1550 \mathrm{~nm}$ [24]. Numerous optomechanical sensing concepts such as freestanding sensor foils with embedded EpoCore waveguide grating structures [25] or flexible carrier substrates with one-side EpoCore strip waveguides for $1550 \mathrm{~nm}$ [26] and $850 \mathrm{~nm}$ operation [27] have been demonstrated. EpoCore Bragg gratings, furthermore, maintain attractive potential for integrated optical sensing in low-cost biochemical lab-on-a-chip applications [28], not least because of their proven biocompatibility to human blood [29]. In this context, a substantially enhanced refractive index sensitivity of EpoCore Bragg gratings was recently attained by high-refractive $\mathrm{TiO}_{2}$ coatings empowering biomedical sensing applications [30].

In this work, we comprehensively investigate the formation and optimization of EpoCore polymer waveguide Bragg gratings by direct excimer laser deep UV irradiation through $+1 /-1$ phase masks and demonstrate promising basic applications for biomedical rapid-testing purposes. Generally, a simple Bragg grating element is formed by a uniform periodic modulation of a waveguide's refractive index (RI), leading to a spectral narrowband reflection signal at the constructively interfering Bragg wavelength $\lambda_{B}$ according to:

$$
m \lambda_{B}=2 n_{\text {eff }} \Lambda
$$

Thereby, the effective refractive index of the supported optical waveguide mode is represented by $n_{\text {eff }}$, while the grating period is denoted by $\Lambda$, whereas $m$ describes the integer order of the Bragg grating reflection [31]. The spectral position of $\lambda_{B}$ is consequently influenced by external parameters such as temperature or the surrounding refractive index (SRI) acting on the waveguide grating structure, either changing $n_{\text {eff }}$ or $\Lambda$. Hence, a respectively induced Bragg wavelength shift represents the Bragg grating's sensor signal. The structure's maximum reflectivity $R_{\max }$ at $\lambda_{B}$ is predominantly dictated by the grating's RI contrast $\Delta n_{B G}$ and the grating length $l_{B G}$ after:

$$
R_{\text {max }}=\tanh ^{2}\left(\frac{\pi}{\lambda_{B}} \Delta n_{B G} M_{p} l_{B G}\right)
$$

whereas the fractional modal overlap $M_{p}$ with the grating dimensions is given by the waveguide design. Moreover, the full-width-at-half-maximum (FWHM) of the reflection signal is defined by:

$$
\Delta \lambda_{F W H M}=\lambda_{B} S \sqrt{\left(\frac{\Delta n_{B G}}{2 \bar{n}}\right)^{2}+\left(\frac{1}{N}\right)^{2}}
$$

with the mean grating RI $\bar{n}$ and the number of grating planes $N$. A further reflectivity factor ranges from $S \approx 0.5$ for weak gratings to $S \approx 1$ for strong gratings [32]. Therefore, the resulting Bragg reflection peak is highly dependent on the chosen grating fabrication technology in combination with the employed waveguide material's modifiability. An optimization of $\Delta n_{B G}$ and $l_{B G}$ within the fabrication limits of the applied phase mask 
technique in this work is thus decisive for obtaining optimum peak tracking capability of strong, narrowband Bragg sensor signals. Carried-out investigations on Bragg grating formation in EpoCore are supported by comprehensive optical grating and waveguide simulations. Finally, a reliable and highly stable sensor performance of optimized compact EpoCore-based devices is demonstrated for rapid biomedical refractive index characterizations of whole blood samples along with the detection of human serum albumin solutions by antigen-antibody-reactions on the sensor surface.

\section{Deep UV Photosensitivity of EpoCore Thin Films}

The ability to locally alter the refractive index of an optical material to an adequate amount by specific optical irradiation represents a most vital property for both the fabrication of FBG and PBG concepts. This so-called photosensitivity describes RI changes due to photochemical reactions and subsequent material densification or decomposition triggered either by UV or two-photon absorption mechanisms [33]. In order to quantify the degree of $248 \mathrm{~nm}$ deep UV-induced refractive index modifications of cross-linked EpoCore epoxy material by the use of $\mathrm{m}$-line spectroscopy, unstructured thin films with a thickness of $4 \mu \mathrm{m}$ were prepared by spin coating EpoCore 5 photoresist solution at $3000 \mathrm{rpm}$ for $60 \mathrm{~s}$ on standard 3" silicon wafers. After a 5-min soft bake at $90{ }^{\circ} \mathrm{C}$, a thorough irradiation step with $200 \mathrm{~mJ} / \mathrm{cm}^{2}$ of $365 \mathrm{~nm}$ mercury arc lamp emission using a standard mask alignment system (EV Group EVG620) in flood exposure mode initiated photochemical reactions inside the flat negative photoresist layers. Consequently, a 5-min-long hot plate treatment at $85{ }^{\circ} \mathrm{C}$ crosslinked the exposed epoxy thin films. Afterwards, the silicon wafers were diced into $12 \mathrm{~mm} \times 12 \mathrm{~mm}$ samples for subsequent $248 \mathrm{~nm} \mathrm{KrF}$ excimer laser illumination (Coherent BraggStar S-Industrial). Thereby, the laser's output beam was homogenized and adjusted to sample size employing a micro lens array resulting in a single-pulse laser fluence of $1 \mathrm{~mJ} / \mathrm{cm}^{2}$. The EpoCore samples were then exposed to a total laser fluence ranging from $0.5 \mathrm{~J} / \mathrm{cm}^{2}$ to $50 \mathrm{~J} / \mathrm{cm}^{2}$ corresponding to 500 and 50.000 laser pulses respectively. The induced refractive index change, i.e., the photosensitivity of EpoCore was characterized by a prism coupler m-line spectrometer (Metricon 2010/M) at the working wavelength of $1548.3 \mathrm{~nm}$ directly after laser illumination and after tempering all samples for $1 \mathrm{~h}$ at $100{ }^{\circ} \mathrm{C}$. Furthermore, the samples were remeasured after storing the samples in a dark and dry environment for 5 months and after another temper step for $12 \mathrm{~h}$ at $50^{\circ} \mathrm{C}$ as depicted in Figure 1. A positive and non-linear index modification with a maximum amount of $\Delta n=(1.8 \pm 0.2) \times 10^{-3}$ was observed, which remained highly stable even after thermal aging and long-term storage procedures. All four measured sample conditions exhibited a similarly strong RI increase at low-total laser fluences up to $10 \mathrm{~J} / \mathrm{cm}^{2}$.

Above this threshold value, further RI increase moderated to a notably smaller amount. No thermal or temporal degradation of $\Delta n$ was observed. Therefore, these experimental results revealed a significant stability of the induced RI modifications representing an imperative property for robust and reliable epoxy Bragg grating sensor elements in practical use. Moreover, the initially steep RI modification curve of the epoxy polymer at lower UV doses offers a wide scope for the design of both weakly and strongly reflecting Bragg grating elements. 


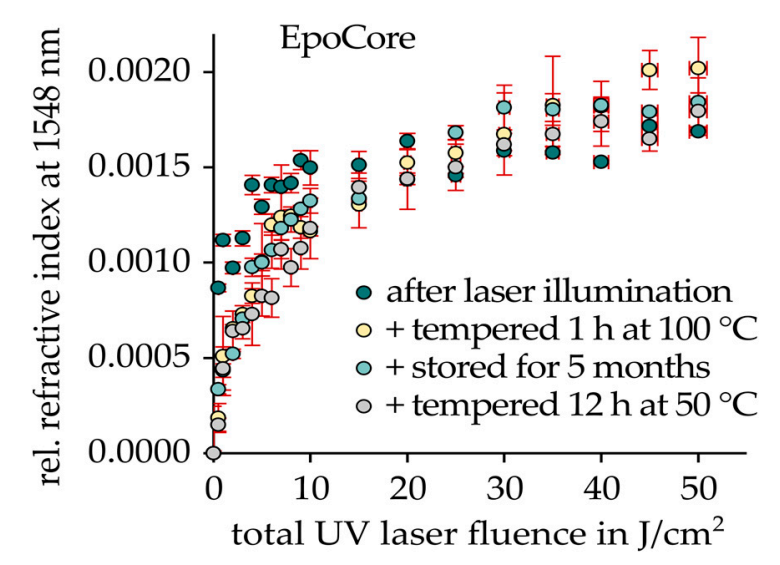

(a)

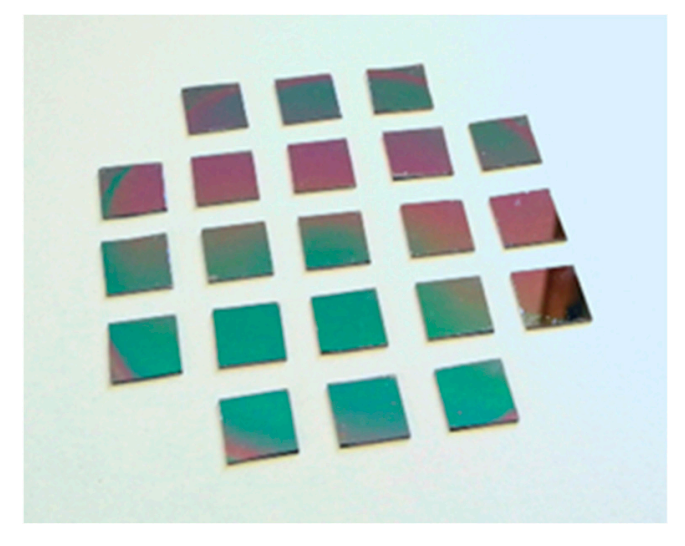

(b)

Figure 1. Influence of total excimer laser fluence on refractive index change of crosslinked EpoCore thin films on silicon samples. (a) Thermal and temporal aging steps reveal a high refractive index modification stability. (b) Photography of the $(12 \times 12) \mathrm{mm}^{2}$ EpoCore on silicon samples.

\section{Fabrication of Epoxy Waveguide Bragg Gratings}

\subsection{Photolithography Process}

Figure 2 provides an overview of the required main steps of the photolithography process for the fabrication of precisely structured all-polymer waveguide Bragg gratings. At first, a cleaned Topas 6017 COC substrate (a) is prepared by washing polymer sheets with a thickness of $1 \mathrm{~mm}$ in acetone and isopropyl alcohol (IPA). After blow drying with nitrogen, the polymer surface is activated by $\mathrm{O}_{2}$ plasma treatment (Diener Femto), improving wettability and adhesion of subsequently spin coated EpoClad 10 photoresist solution at $3000 \mathrm{rpm}$ for an approximate thickness of $10 \mu \mathrm{m}(\mathrm{b})$. The formed layer is soft baked for $5 \mathrm{~min}$ at $120^{\circ} \mathrm{C}$ on a hotplate before $365 \mathrm{~nm}$ UV flood exposure with a total dose of $350 \mathrm{~mJ} / \mathrm{cm}^{2}$ (c). The EpoClad film as an optional waveguide under-cladding is finished by a post-exposure-bake for $5 \mathrm{~min}$ at $120^{\circ} \mathrm{C}$. The fabrication of the functional waveguide Bragg grating elements is in turn initiated by $\mathrm{O}_{2}$ plasma activation of the present polymer surface. The EpoCore photoresist solution is spin coated with a precisely chosen rotational speed to achieve the desired waveguide thickness (d). After soft baking for $5 \mathrm{~min}$ at $90^{\circ} \mathrm{C}$, the EpoCore layer is UV exposed with $200 \mathrm{~mJ} / \mathrm{cm}^{2}$ at $365 \mathrm{~nm}$ through an amplitude mask in contact mode defining waveguide widths of $10 \mu \mathrm{m}$ and $15 \mu \mathrm{m}$ (e).

Again, a post-exposure-bake for $5 \mathrm{~min}$ at $85{ }^{\circ} \mathrm{C}$ crosslinks the illuminated epoxy material, leaving behind the optical strip waveguide with a rectangular cross-section after selectively dissolving unirradiated EpoCore in mrDev600 (micro resist technology) developer bath for $60 \mathrm{~s}(\mathrm{f})$. Bragg gratings are locally inscribed into the waveguides via $248 \mathrm{~nm}$ $\mathrm{KrF}$ excimer laser irradiation at $20 \mathrm{~Hz}$ through $+1 /-1$-phase masks (Ibsen Photonics) with grating periods around $1000 \mathrm{~nm}$ and maximum grating lengths of $10 \mathrm{~mm}(\mathrm{~g})$.

To create a further, symmetrical upper-cladding layer on top of the waveguide structures, another EpoClad layer is processed equally to the under-cladding scheme (h). However, employed amplitude masks during the $365 \mathrm{~nm}$ exposure step enable a precise microstructuring of this cladding layer as well (i). Since EpoClad still exhibits a sticky surface after soft bake, UV exposure must be performed in proximity mode. Fabrication is completed by developing the thus defined EpoClad structures for $180 \mathrm{~s}$ in mrDev600 (j). Finally, a manual permanent optical fiber coupling to the mechanically polished waveguide facet using optical adhesive NOA76 (Norland) enables standard interrogation (Micron Optics sm125) of the Bragg grating elements around $1550 \mathrm{~nm}$. Despite inevitably introduced coupling losses in dependence of the fabricated waveguide's dimensions, a sufficiently strong and highly stable Bragg reflection monitoring is still provided by accurate central 
and close coupling of the waveguide facets. The formed optical interconnection remains reliable in the long term under normal room and experimental handling conditions.

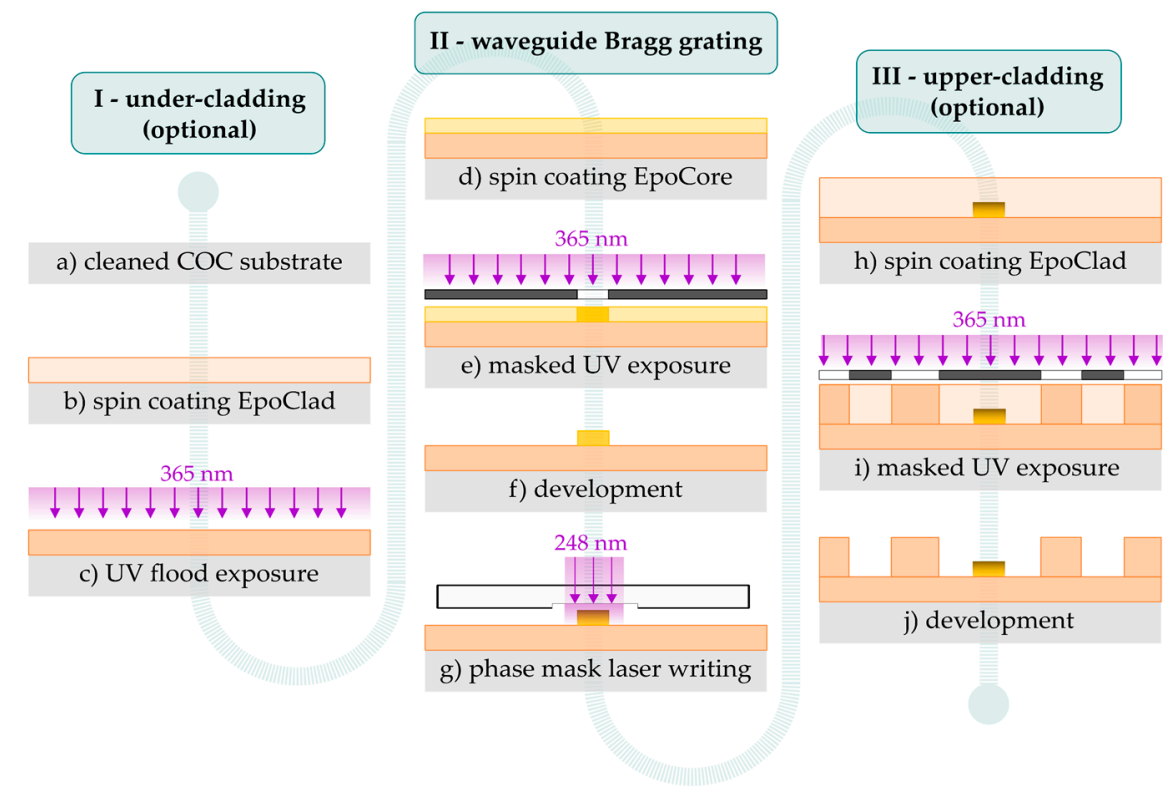

Figure 2. Required photolithography process steps for the fabrication of epoxy-based waveguide Bragg grating sensors.

\subsection{Phase Mask Simulation and Bragg Grating Inscription}

The employed transmission +1 / -1 -phase mask design for direct Bragg grating inscription is intended to use the +1 and -1 diffraction orders only to produce an ideal sinusoidal near-field interference pattern for the specific laser wavelength of $248 \mathrm{~nm}$. However, real phase masks are flawed by non-vanishing amounts of zero order and higher order diffraction. Diffraction efficiency is thereby mainly dictated by the etching depth of the grooves in the fused silica block surface forming the optical grating. An optical diffraction parameter run simulation (LightTrans VirtualLab) for a grating period of $1053 \mathrm{~nm}(50 \%$ duty cycle) was performed to identify the optimum grating depth of $265 \mathrm{~nm}$ yielding maximum $+1 /-1$ order efficiency of $\sim 37 \%$ and minimum zero and higher order efficiencies $<1 \%$. Furthermore, the corresponding near-field interference pattern was simulated in Figure 3 revealing distance-dependent occurrence of the intensity maxima. Three characteristic intensity cross-sections illustrate possibly appearing grating formations in the photosensitive epoxy material, producing either a dominant Bragg grating period equal to the phase mask's period $\Lambda_{\mathrm{pm}}$ or $\Lambda_{\mathrm{pm}} / 2$. Thereby, the phase mask's precise orientation as well as the waveguide thickness and the $248 \mathrm{~nm}$ penetration depth in EpoCore represent critical Bragg grating fabrication parameters. However, according to equation 1 with respectively applied diffraction order $\mathrm{m}$, both types of induced grating periods result in an identical Bragg wavelength at the desired working wavelength. Despite its non-ideal characteristics, the phase mask technique is thus a robust and reliable tool for direct laser inscription of Bragg grating elements.

In Figure 4, the distinctive influence of the totally impinged deep UV fluence on the phase mask assisted formation of EpoCore waveguide Bragg gratings is displayed. The irradiated EpoCore waveguides (marked in yellow) with a thickness of $4 \mu \mathrm{m}$ were investigated by differential interference contrast (DIC) light microscopy and laser scanning microscopy (LSM). In the lower UV dose regime $\leq 10 \mathrm{~J} / \mathrm{cm}^{2}$, grating types with a characteristic grating period of $\Lambda_{\mathrm{pm}}$ were inscribed and exhibited depth-dependent $\pi$-shifts of the grating structure (see waveguide top view micrograph in Figure 4a) in excellent accordance with the described near-field simulation pattern of a real phase mask in Figure 3. Consequently, only strong primary intensity maxima of the phase mask near-field pattern 
contribute to an observable RI modification of the EpoCore material, while secondary maxima evidently fall below a modification threshold.

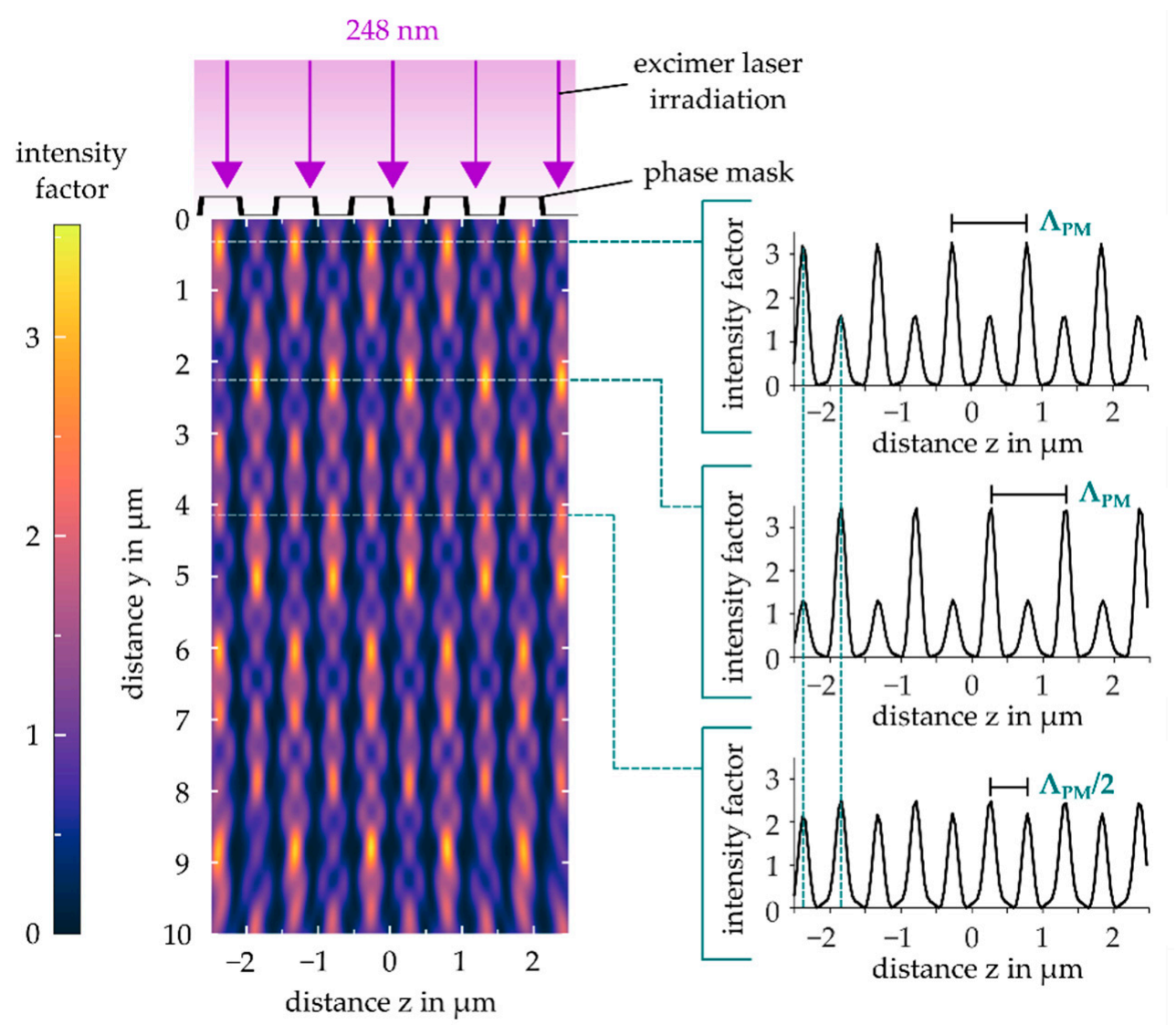

Figure 3. Simulated phase mask near-field (using Lighttrans VirtualLab) for a grating period of $1053 \mathrm{~nm}$ and an optimized grating depth of $265 \mathrm{~nm}$.

However, applying significantly higher deep UV doses $>10 \mathrm{~J} / \mathrm{cm}^{2}$ result in modification contribution of the weaker secondary intensity maxima as well. In this case, Bragg gratings with a grating period of $\Lambda_{\mathrm{pm}} / 2$ are fabricated in the epoxy bulk location directly below the surface (see cross sectional waveguide micrograph in Figure $4 \mathrm{~b}$ ). A comparative grating formation behavior was confirmed in photosensitive PMMA bulk polymer, resembling the intensity maxima of the phase mask near-field pattern in a similar way. A strongly decreasing modification strength with increasing material depth was observable, which corresponds to the material-dependent penetration depth of the $248 \mathrm{~nm}$ laser pulses. Moreover, an additional grating structure with a grating period of $\Lambda_{\mathrm{pm}}$ was implied at the surface, indicating occurring material ablation by the highly dosed laser irradiation.

Employing surface topography analysis by LSM, both irradiation cases of epoxy waveguides were investigated regarding surface roughness. A total UV laser fluence of $10 \mathrm{~J} / \mathrm{cm}^{2}$ did not cause a measurable surface corrugation in comparison with unirradiated EpoCore surfaces. In consequence, the fabricated Bragg gratings act as pure refractive index gratings inside the epoxy waveguide. In contrast, a much higher deep UV dose of $40 \mathrm{~J} / \mathrm{cm}^{2}$ induced additional periodical surface indentations with a grating period of $\Lambda_{\mathrm{pm}}$ due to laser ablation at the high-intensity maxima in the near-field pattern. The Bragg reflections of these kind of waveguide structures therefore result from a combination of the formed surface and bulk grating parts with different periods. 
(a) low UV dose scenario

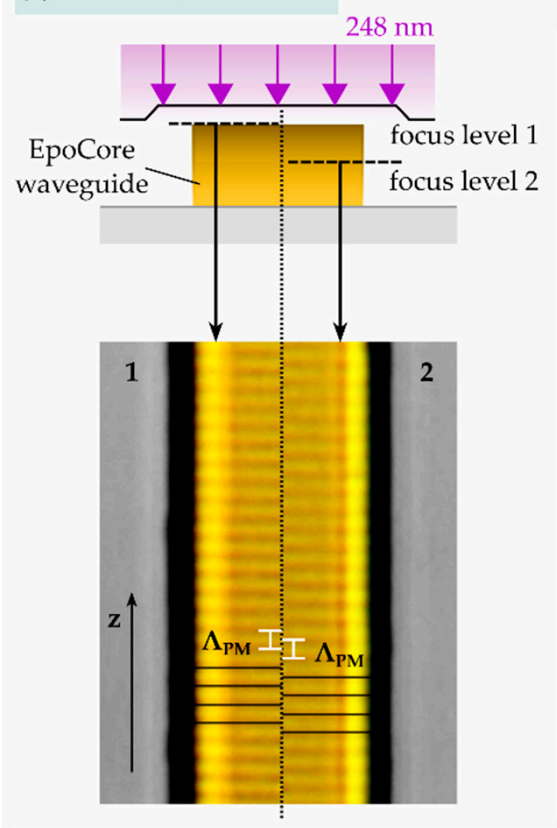

(c) surface roughness of EpoCore waveguide

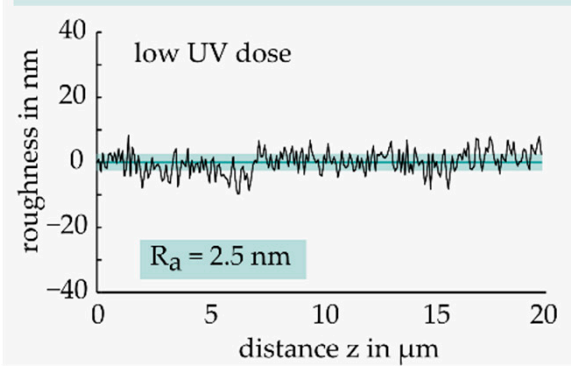

(b) high UV dose scenario

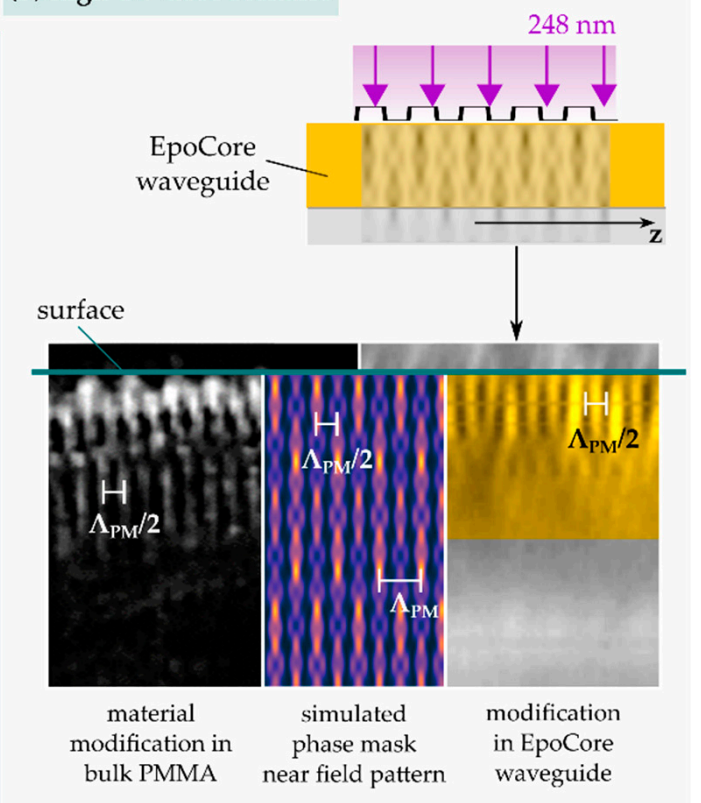

Figure 4. Bragg grating formation: comparison of low $\left(10 \mathrm{~J} / \mathrm{cm}^{2}\right)$ and high $\left(40 \mathrm{~J} / \mathrm{cm}^{2}\right) \mathrm{UV}$ dose effects on surface and bulk volume of EpoCore waveguides irradiated through a phase mask.

\section{Online Monitoring of Bragg Signal Formation}

To identify optimum excimer laser parameters for the inscription of Bragg gratings in EpoCore, $50 \mathrm{~mm}$ long waveguides (15 $\mu \mathrm{m}$ width, $5 \mu \mathrm{m}$ height) were prepared on an EpoClad layer as depicted in Figure 5. At the waveguide end, a short $4 \mathrm{~mm}$ long reference grating $(\Lambda=990 \mathrm{~nm})$ encapsuled in EpoClad ensured a permanently attached fiber-to-chip coupling position, providing a high fundamental mode Bragg signal quality. An onlinemonitored Bragg grating was inscribed with increasing total laser irradiation into the waveguide center using a $+1 /-1$-phase mask with $\Lambda_{\mathrm{pm}}=1008 \mathrm{~nm}$ and a grating length of $10 \mathrm{~mm}$. The successively rising reflection peak was characterized in terms of peak strength, FWHM, peak position as well as Bragg grating attenuation, i.e., the grating insertion loss, which is determined by decreasing peak strength of the reference grating.

The results of the parameter tracking procedure are summarized in the diagrams of Figure 6. At a low total laser fluence of $2 \mathrm{~J} / \mathrm{cm}^{2}$, a symmetric Bragg reflection peak around $1576.2 \mathrm{~nm}$ already appeared. With ongoing irradiation, an initially steep increase of the Bragg peak height occurred until $5 \mathrm{~J} / \mathrm{cm}^{2}$, which eventually saturated around $10 \mathrm{~J} / \mathrm{cm}^{2}$. FWHM showed a similar behavior, indicating an increasing grating contrast $\Delta n$ of the forming Bragg structure with a constant length of $10 \mathrm{~mm}$. BG attenuation soared up quickly, even at a very weak UV impact to a stable $0.19 \mathrm{~dB} / \mathrm{mm}$ up to an applied fluence of $10 \mathrm{~J} / \mathrm{cm}^{2}$. Meanwhile, the peak position and thus the effective refractive index rose continuously, suggesting an offset-like average refractive index increase of the whole irradiated EpoCore structure, i.e., an elevated mean grating RI $\bar{n}$. This tendency 
together with the unchanging peak strength, however, implies a persistent grating contrast. Considering the delayed but growing influence of the less intense secondary maxima from the near-field pattern (Figure 3) by means of the found EpoCore RI modification curve (Figure 1), a constantly lasting grating contrast is explicable. By exceeding a total laser fluence of $10 \mathrm{~J} / \mathrm{cm}^{2}$, a significantly increasing BG attenuation was observable due to the beginning ablation mechanisms. Additional surface gratings were formed, leading to extra scatter loss. Furthermore, the Bragg reflection progressively tended to split into several peaks evoking a rising FWHM as well.
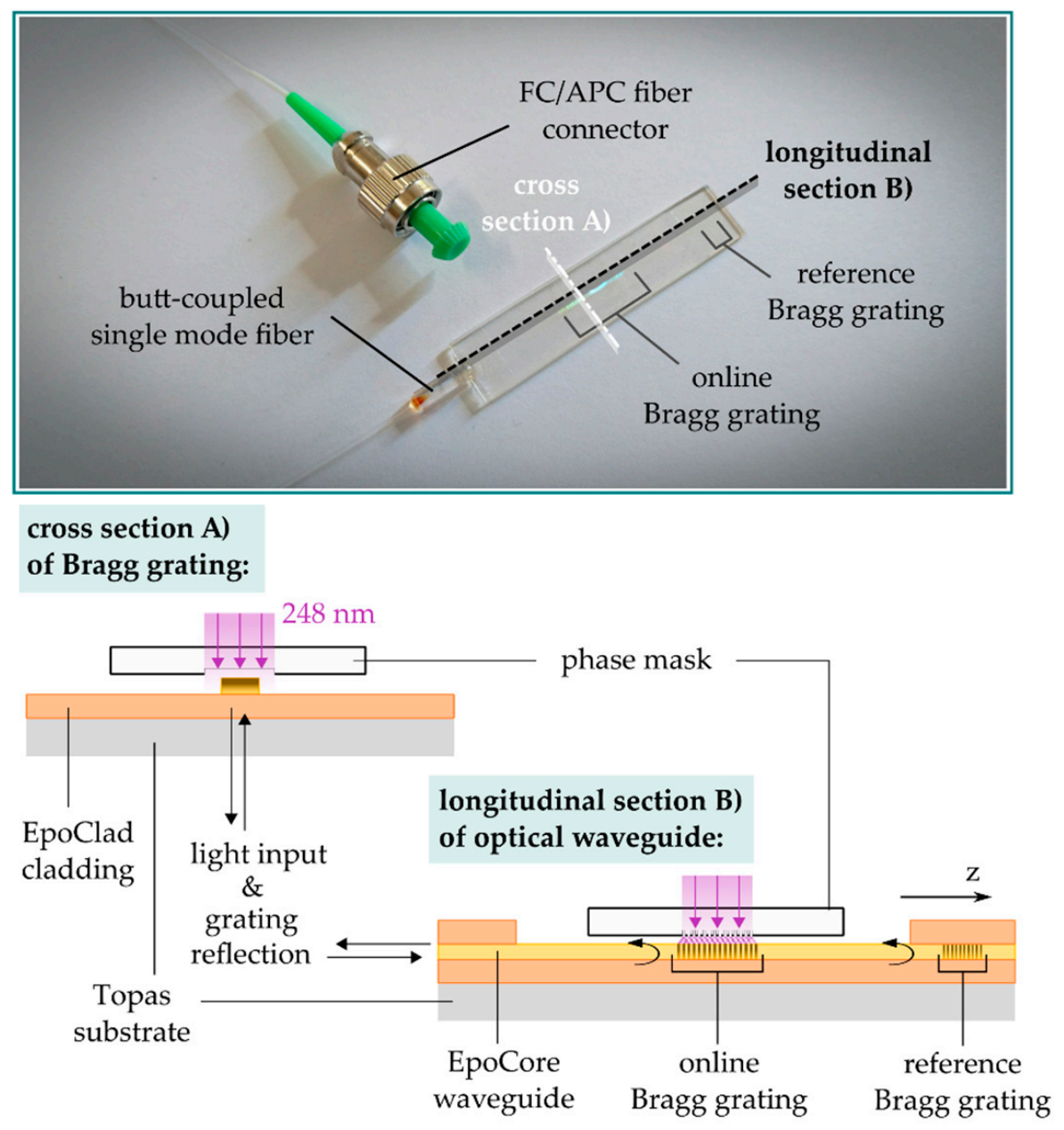

Figure 5. Schematic for monitoring the reflection signal behavior of stepwise deep UV induced Bragg gratings in EpoCore waveguides.

Therefore, the total laser fluence employed for grating formation can be divided into two parts. In the lower dose regime of up to $10 \mathrm{~J} / \mathrm{cm}^{2}$, type I gratings (marked in green), i.e., refractive index gratings in the EpoCore material were fabricated. Above $10 \mathrm{~J} / \mathrm{cm}^{2}$, type I as well as type II gratings, i.e., surface damage gratings induced by ablation were formed (marked in grey). Taking all evaluated parameters into account, a total UV laser fluence of $10 \mathrm{~J} / \mathrm{cm}^{2}$ is proposed for inscribing low-loss Bragg gratings with strong and narrow-band reflection signals. 

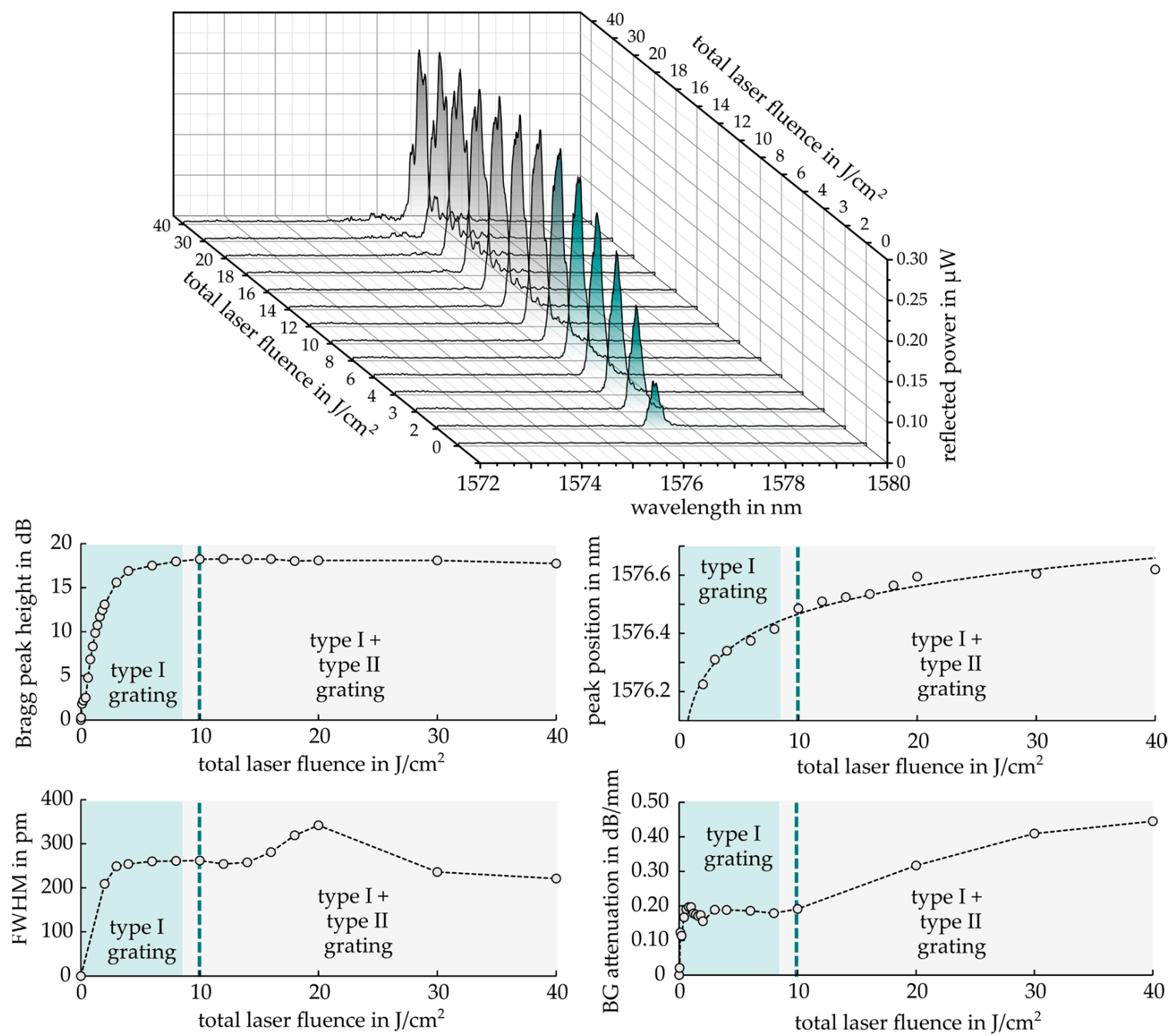

Figure 6. Bragg grating signal formation due to ongoing excimer laser irradiation.

\section{Comparison of Measured and Simulated Bragg Reflections}

To estimate the attainable phase mask assisted grating contrast in EpoCore, which mainly determines maximum grating reflectivity and FWHM, comprehensive optical grating simulations in strip waveguides were carried out using RSoft GratingMOD software. In Figure 7 , the resulting influence of the grating length $l_{B G}(1$ to $10 \mathrm{~mm})$ as well as the influence of the grating contrast $\left(0\right.$ to $\left.5 \times 10^{-4}\right)$ on maximum reflectivity and FWHM is summarized in terms of heat maps. Apparently, strong Bragg reflections principally occur with a grating contrast above $1 \times 10^{-4}$ at long grating length. However, a higher grating contrast allowed for shorter gratings of several $\mathrm{mm}$ only to reach comparable Bragg reflection strength.

In turn, the FWHM significantly increases in the case of a short, strong grating design. Hence, for precisely detectable, sufficiently narrow EpoCore Bragg grating signals for sensor purposes, minimum grating lengths $l_{B G} \geq 2 \mathrm{~mm}$ are advised.

By fitting the measured FWHM data from Figure $6\left(l_{B G}=10 \mathrm{~mm}\right)$ with the simulated heatmap data in Figure 7, a maximum grating contrast of $\Delta n_{\mathrm{BG}}=2.8 \times 10^{-4}$ was determined. The results of a real grating length experiment ( $1 \mathrm{~mm}$ to $8 \mathrm{~mm}$ in steps of $1 \mathrm{~mm}$ ) with $\Lambda_{\mathrm{pm}}=990 \mathrm{~nm}$ and a total laser fluence of $8 \mathrm{~J} / \mathrm{cm}^{2}$ are shown in Figure 8 . By measuring 
the strength of the respective spectral transmission power dips $T_{d}$ (in $\mathrm{dB}$ ) at the Bragg wavelength, maximum grating reflectivity was calculated by:

$$
R_{\max }=1-10^{\frac{-T_{d}}{10}}
$$

(a) maximum reflectivity

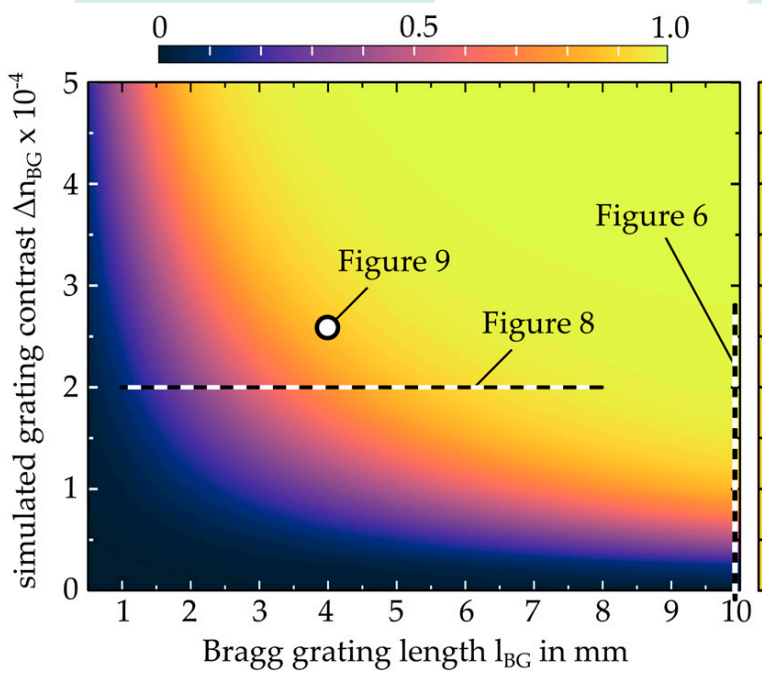

(b) FWHM in nm

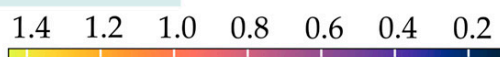

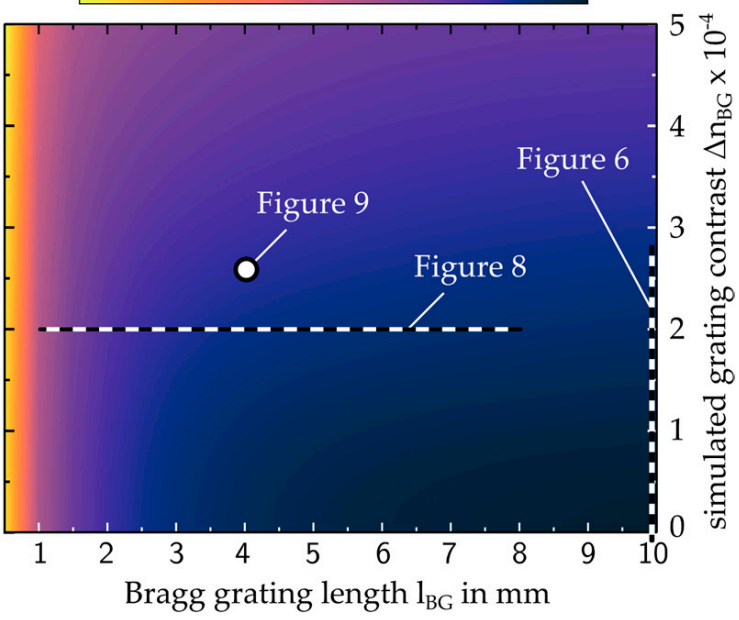

Figure 7. Simulated maximum reflectivity and full-width-at-half-maximum values (using RSoft GratingMOD) and the corresponding positions of carried out measurements.

(a)

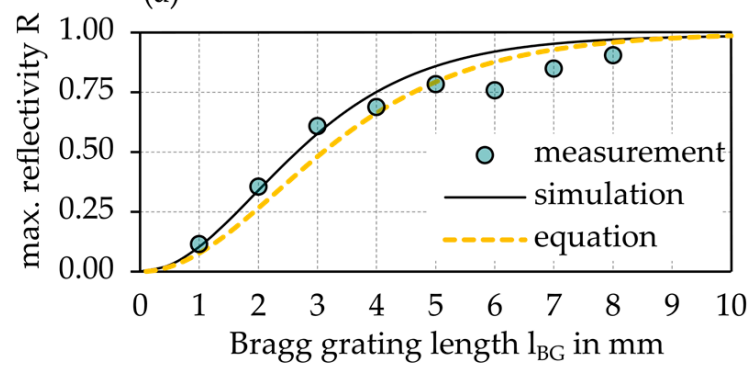

(c)

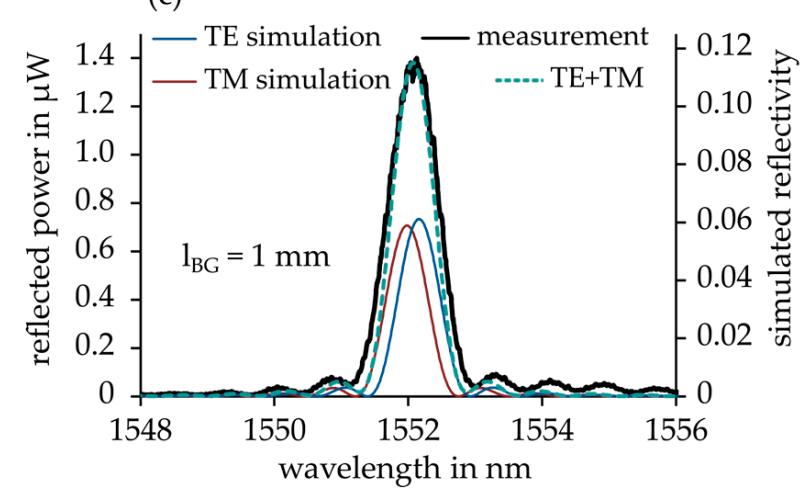

(b)

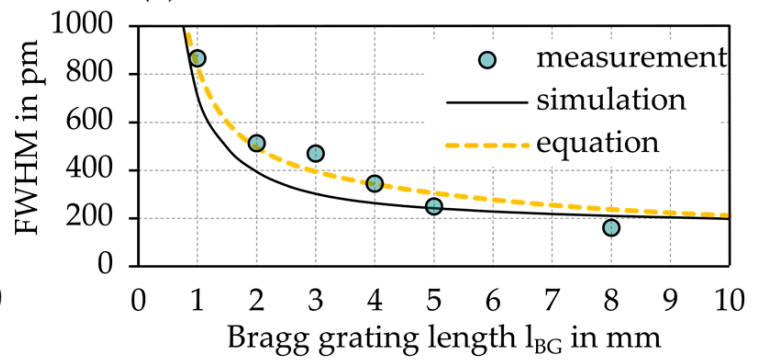

(d)

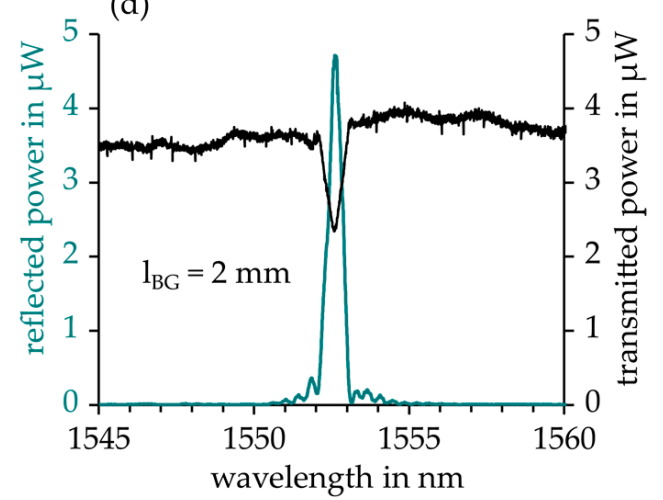

Figure 8. Influence of grating length on (a) maximum reflectivity and (b) the full-width-at-half-maximum (FWHM). Measurements are in good agreement with simulation data and theoretical equation. (c) compares simulated and measured peak appearance for a grating length of $1 \mathrm{~mm}$. (d) exemplifies the determination of maximum grating reflection for a grating length of $2 \mathrm{~mm}$. 
Figure 8a,b show grating length dependent $R_{\max }$ und FWHM measurement results, which conform well to the optical simulation data and theoretical parameter equations 2 and 3 (yellow curves). In Figure 8c, the measured Bragg peak for a grating length of $1 \mathrm{~mm}$ is compared to simulated data for fundamental transverse electric (TE) and transverse magnetic (TM) mode reflection of the according Bragg structure which compose the overall resulting Bragg reflection. Again, an extraordinary agreement to the optical simulation result was found. Figure $8 \mathrm{~d}$ exemplifies the measurement of the transmission dip at the Bragg wavelength for a grating length of $2 \mathrm{~mm}$. The measured parameters correspond to an achieved grating contrast of $\Delta n_{\mathrm{BG}}=2.0 \times 10^{-4}$ in Figure 7 .

In Figure 9, a representative appearance of a strong $\left(R_{\max }=87 \%\right)$ and narrow-band $(\mathrm{FWHM}=310 \mathrm{~nm})$ Bragg reflection is depicted for optimized fabrication parameters of $\mathrm{F}=10 \mathrm{~J} / \mathrm{cm}^{2}$ and $l_{B G}=4 \mathrm{~mm}$, which is utilized for EpoCore-based Bragg grating sensor applications. The characteristic Bragg reflection relates to an overall grating contrast of $\Delta n_{\mathrm{BG}}=2.6 \times 10^{-4}$ as shown in Figure 7 .

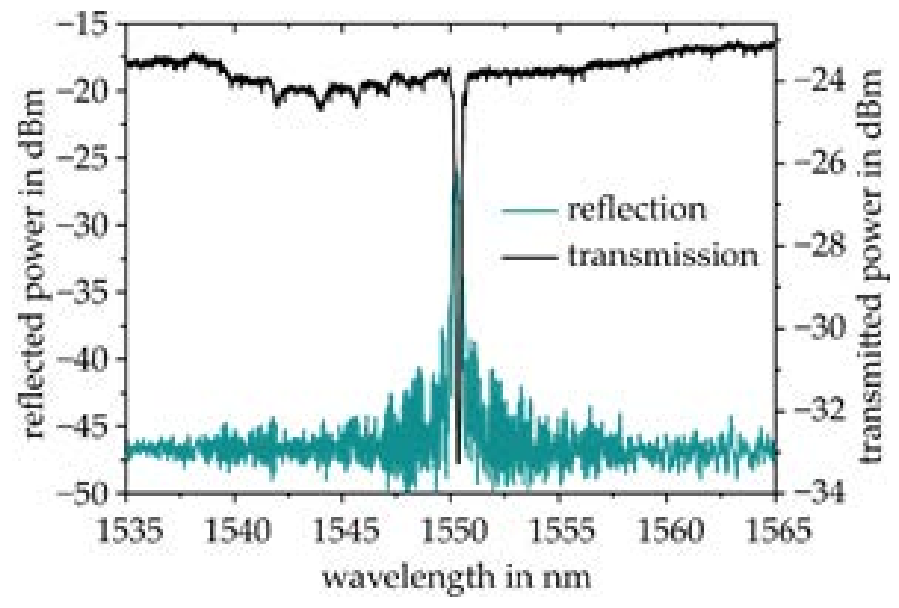

Figure 9. Optimized reflectivity of $\sim 87 \%$ for a $4 \mathrm{~mm}$ short epoxy waveguide Bragg grating sensor with low insertion loss.

\section{Biomedical Application Potentials of Epoxy-Based Bragg Grating Sensors}

Due to the high stability of crosslinked EpoCore material and its validated hemocompatibility [29], the optical epoxy polymer structures generally offer great potential in biomedical sensing. To employ the polymer waveguide Bragg gratings as efficient refractive index sensors, particular surrounding RI sensitivity-enhancing modifications of the waveguide layout are applied while maintaining the found optimum grating fabrication parameters. First, the waveguide height on Topas 6017 substrate is reduced to $\leq 1 \mu \mathrm{m}$ near the fundamental mode cut-off (as a consequence of the asymmetric waveguide design). Furthermore, a thin high-index $\mathrm{TiO}_{2}$ coating of about $75 \mathrm{~nm}$ is sputtered onto the waveguide Bragg grating surface, as described in detail in [30], to increase evanescent wave portions in the sample media.

In a first application, a thus-modified epoxy Bragg sensor chip is demonstrated as a bulk refractive index sensor for the characterization of whole blood samples in Figure 10.

Additionally, for this particular use, a successful transfer of the former fabrication findings at $1550 \mathrm{~nm}$ to a working wavelength around $1310 \mathrm{~nm}$ is shown. A broadband light source (Thorlabs S5FC1021S SLED) in combination with a circulator and spectrometer (Ibsen I-MON 512) served as interrogation system for sensor chips with grating periods of $840 \mathrm{~nm}$. A highly reproducible sensor calibration curve with remarkable Bragg wavelength shifts of up to $65.9 \mathrm{~nm}$ was recorded using Cargille index fluids in the RI range 1.2964-1.5137 at $1310 \mathrm{~nm}$ on top of the sensor grating area. Likewise, a characterization of sugar solutions verified reliable sensor performance by revealing a beneficial resistance against moisture influence. SRI sensitivity amounted to $195 \mathrm{~nm} / \mathrm{RIU}$ for $\mathrm{n}=1.4$ and $295 \mathrm{~nm} / \mathrm{RIU}$ for $\mathrm{n}=1.5$. By this method, three droplets of whole blood were classified to individual refractive indices 
of $1.3366,1.3386$, and 1.3325 , respectively. The accurately determined RI of whole blood or blood plasma potentially serves as a general health indicator.

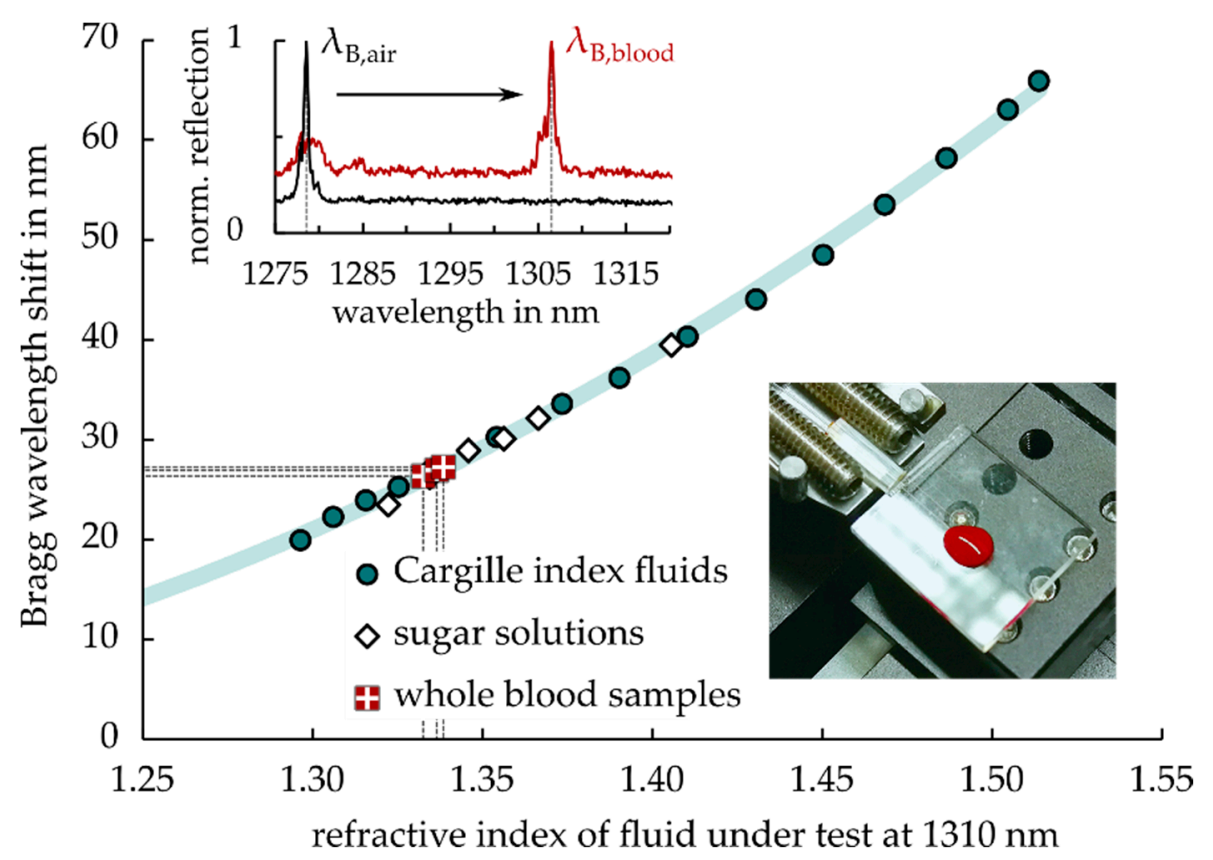

Figure 10. Refractive index measurement of whole blood samples using sensitized epoxy-based Bragg grating sensors. The inset exemplifies monitored Bragg wavelength shifts due to blood droplet exposure.

Commonly, for a selective detection and quantification of individual blood and blood plasma components, highly specific binding layers are mandatory. To qualify the employed epoxy Bragg grating sensors for basic biomedical screening purposes, selective binding of essential human serum albumin (HSA) protein to the optical sensor's surface via antigenantibody-reaction was evaluated. For this purpose, the Bragg grating areas were uniformly exposed to a highly concentrated $(360 \mu \mathrm{g} / \mathrm{mL}$ ) anti-HSA antibody (DuoSet ELISA DY1455, R\&D Systems, Minneapolis, MN, USA) solution in phosphate buffered saline (PBS) for 45 min. By intense washing with a buffer solution $\left(0.05 \%\right.$ Tween ${ }^{\circledR} 20$ in PBS, Sigma Aldrich Chemie $\mathrm{GmbH}$, Taufkirchen, Germany), all residual unbound antibodies were removed efficiently. After gently drying the sensor samples by nitrogen purge, a blocking step was introduced by exposing the sensor surfaces to bovine serum albumin (BSA) solution $(0.1 \%$ BSA in PBS) for $30 \mathrm{~min}$. Thereby, BSA serves to passivate the unoccupied and hence unspecific binding-sites of the waveguide grating surfaces and does not corrupt the functional antibody coating. After repeated washing and drying, the epoxy-based Bragg grating sensors were prepared for defined exposure to the targeted HSA solutions.

Figure 11 demonstrates the full temporal, characteristic Bragg wavelength behavior during sensor preparation and selective HSA binding. All these sensor signals were monitored by standard interrogation system in the wavelength range around $1550 \mathrm{~nm}$.

Large and instantaneous Bragg wavelength shifts are induced by the bulk refractive index of the applied fluids themselves, while surface binding reactions in general cause a small, gradual alteration of the sensor signal's spectral position until saturation. Thus, for the detection of selective HSA binding and to investigate the influence of HSA concentration, the gradients of the induced Bragg wavelength shifts evoked by different HSA concentrations of $2,5 \mathrm{mg} / \mathrm{mL}, 25 \mathrm{mg} / \mathrm{mL}$, and $50 \mathrm{mg} / \mathrm{mL}$ were evaluated accordingly. Figure 12a shows the HSA concentration-dependent sensor responses in comparison. A distinct and continuous positive Bragg wavelength shift was observed during the exposure of the three different measurand concentrations. After twelve minutes of exposure, all Bragg sensor signals were in a stable condition indicating saturation state. In Figure 12b, the 
Bragg wavelength differences at the well-detectable reference points at $4 \mathrm{~min}$ and $12 \mathrm{~min}$ of HSA exposure were consulted as a sensitivity measure (average of two measurements). The obtained curve resembled the exponential shape sensitivity against the surrounding refractive index of the raw, non-selective epoxy-based Bragg grating sensor. Especially, the experimental results attested to the optical sensor design considerable sensitivity near healthy-state HSA concentrations (33-52 mg/mL) in human blood plasma [34], suggesting highly valuable biomedical rapid test applications.

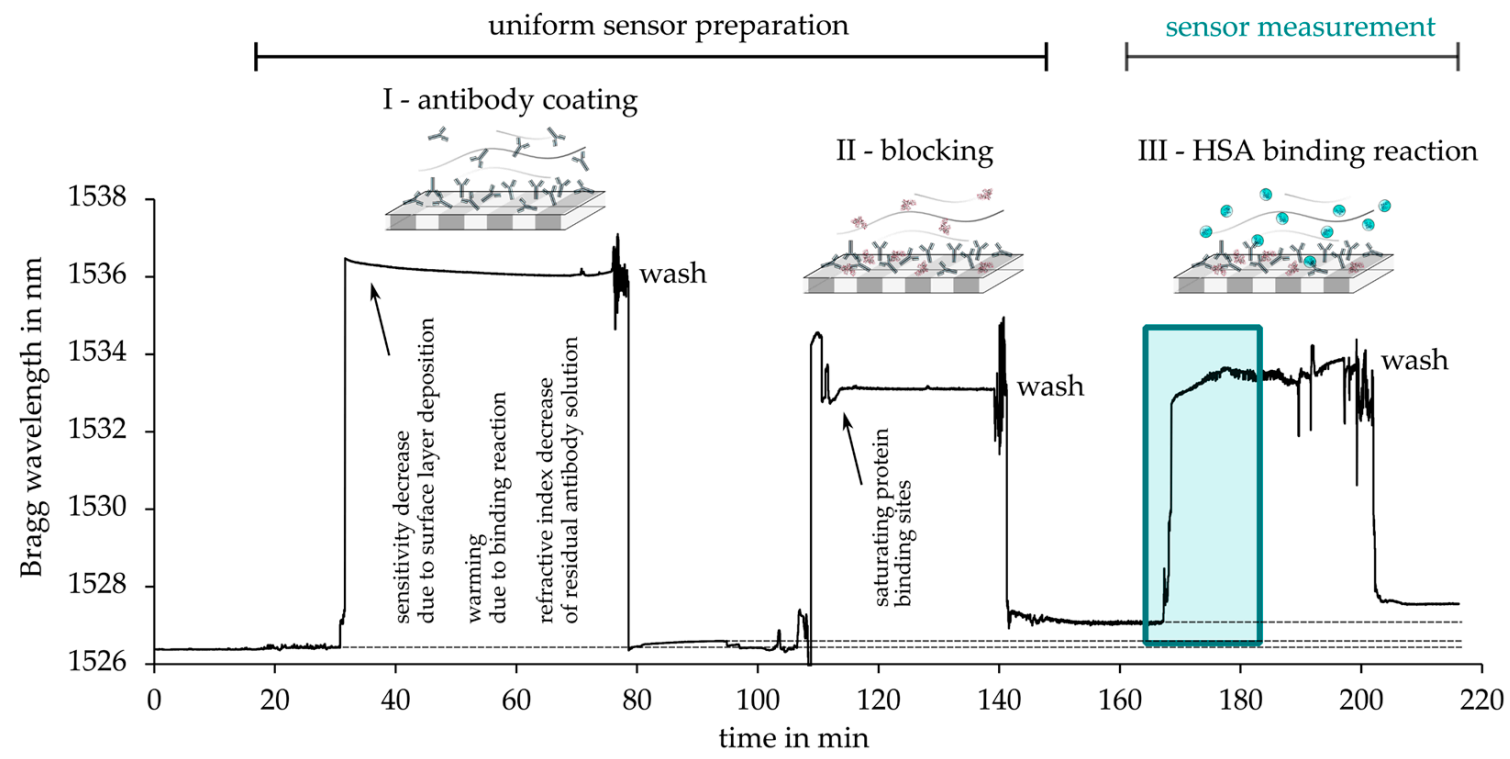

Figure 11. Exemplary full temporal sensor response during antibody-antigen-assay for human serum albumin detection (antigen sensing task is highlighted in green box).

(a)

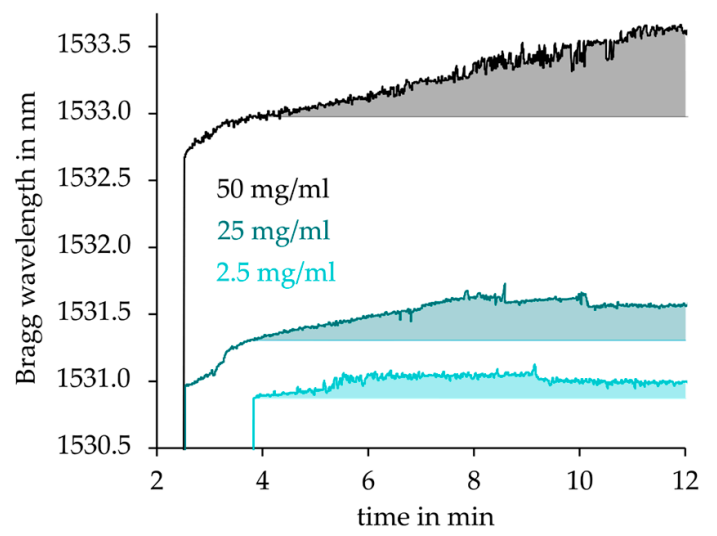

(b)

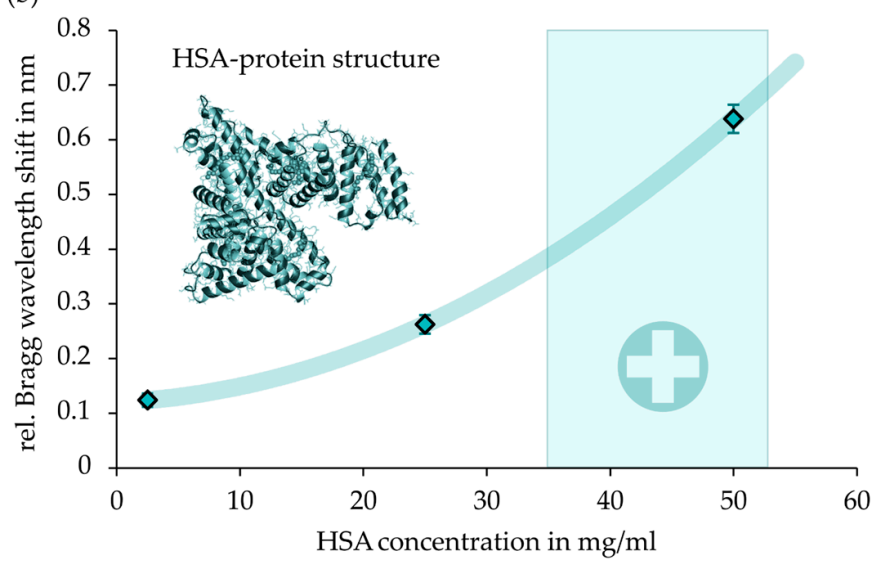

Figure 12. Comparative sensor response in dependence on HSA concentration. (a) temporal sensor response. (b) determined Bragg wavelength shifts.

Since all the employed sensor chips originated from the same fabrication batch (with the same waveguide and coating thickness), very high measurement reproducibility using different sensor chips was obtained throughout the experiments. Moreover, with respective thorough cleaning and drying procedures of the individual sensor surface after each measurement, a high measurement repeatability was given as well. 


\section{Conclusions}

In this work, we extensively investigated the nature of deep UV-induced Bragg grating formation in EpoCore strip waveguides by the use of the popular phase mask technique. The laser fluence dependent refractive index modifications of the epoxy material in combination with the complex near field diffraction patterns of non-ideal phase masks strongly affected the resulting grating type. Optimum grating fabrication parameters for compact, long-term stable, strong, and narrowband Bragg reflections in EpoCore were derived with exceptional agreement to optical grating simulations. Moreover, a modified waveguide grating design based on all preliminary findings was employed for highly sensitive surrounding refractive index determination with sensitivities up to $295 \mathrm{~nm} /$ RIU for sample media with $\mathrm{n}=1.5$. Attractive biomedical application potential for rapid characterization of whole blood samples as well as for the specific determination of human serum albumin were demonstrated successfully. Consequently, an optimized Bragg sensor implementation into future optofluidic lab-on-a-chip devices in combination with innovative, low-cost wavelength interrogation schemes (as for example in [35]) is strongly encouraged.

Author Contributions: Conceptualization, S.H., M.R., H.-D.L., B.S. and R.H.; methodology, S.H., M.R.; software, S.H.; validation, S.H., M.R., H.-D.L., B.S. and R.H.; formal analysis, S.H. and M.R.; investigation, S.H.; resources, R.H., H.-D.L. and M.R.; data curation, S.H. and M.R.; writing-original draft preparation, S.H.; writing-review and editing, S.H., M.R., H.-D.L., B.S. and R.H.; visualization, S.H.; supervision, B.S. and R.H.; project administration, M.R. and S.H.; funding acquisition, S.H., M.R., H.-D.L. and R.H. All authors have read and agreed to the published version of the manuscript.

Funding: This research was funded by the German Bundesministerium für Bildung und Forschung (BMBF), grant number 16ES0811 and by the Bavarian Research Foundation (BFS), grant number AZ-1427-20.

Informed Consent Statement: Informed consent was obtained from all subjects involved in the study.

Data Availability Statement: The data presented in this study are available on request from the corresponding author.

Acknowledgments: We thank Mathias Rommel and Stefan Knopf from Fraunhofer Institute for Integrated Systems and Device Technology IISB for providing sputtered $\mathrm{TiO}_{2}$ coatings.

Conflicts of Interest: The authors declare no conflict of interest.

\section{References}

1. Cusano, A.; Cutolo, A.; Albert, J. Fiber Bragg Grating Sensors. Recent Advancements, Industrial Applications and Market Exploitation; Bentham Science Publishers Ltd.: Sharja, UAE, 2015.

2. Hisham, H.K. Fiber Bragg Grating Sensors: Development and Applications; CRC Press; Taylor \& Francis Group: Boca Raton, FL, USA; London, UK; New York, NY, USA, 2020.

3. Oliveira, R.; Bilro, L.; Nogueira, R. Polymer Optical Fiber Bragg Gratings: Fabrication and Sensing Applications; CRC Press; Taylor \& Francis Group: Boca Raton, FL, USA, 2020.

4. Werneck, M.M.; Nazaré, F.V.B.d.; Allil, R.C.d.S.B. Fiber Bragg Gratings: Theory, Fabrication, and Applications; SPIE Press: Bellingham, WA, USA, 2017.

5. Allwood, G.; Wild, G.; Hinckley, S. Fiber Bragg Grating Sensors for Mainstream Industrial Processes. Electronics 2017, 6, 92. [CrossRef]

6. Ye, X.W.; Su, Y.H.; Han, J.P. Structural health monitoring of civil infrastructure using optical fiber sensing technology: A comprehensive review. Sci. World J. 2014, 652329. [CrossRef]

7. Sahota, J.K.; Gupta, N.; Dhawan, D. Fiber Bragg grating sensors for monitoring of physical parameters: A comprehensive review. Opt. Eng. 2020, 59, 1. [CrossRef]

8. Pevec, S.; Donlagić, D. Multiparameter fiber-optic sensors: A review. Opt. Eng. 2019, 58. [CrossRef]

9. Leal-Junior, A.G.; Diaz, C.A.R.; Avellar, L.M.; Pontes, M.J.; Marques, C.; Frizera, A. Polymer Optical Fiber Sensors in Healthcare Applications: A Comprehensive Review. Sensors 2019, 19, 3156. [CrossRef] [PubMed]

10. Koyama, S.; Haseda, Y.; Ishizawa, H.; Okazaki, F.; Bonefacino, J.; Tam, H.-Y. Measurement of Pulsation Strain at the Fingertip Using a Plastic FBG Sensor. IEEE Sens. J. 2021, 1. [CrossRef]

11. Klimov, N.N.; Mittal, S.; Berger, M.; Ahmed, Z. On-chip silicon waveguide Bragg grating photonic temperature sensor. Opt. Lett. 2015, 40, 3934-3936. [CrossRef] [PubMed] 
12. Sparrow, I.J.G.; Smith, P.G.R.; Emmerson, G.D.; Watts, S.P.; Riziotis, C. Planar Bragg Grating Sensors-Fabrication and Applications: A Review. J. Sens. 2009, 1-12. [CrossRef]

13. Watts, S. Optical microchip sensors. Nat. Photonics 2010, 4, 433-434. [CrossRef]

14. Holmes, C.; Gates, J.C.; Carpenter, L.G.; Rogers, H.L.; Parker, R.M.; Cooper, P.A.; Chaotan, S.; Mahamd Adikan, F.R.; Gawith, C.B.E.; Smith, P.G.R. Direct UV-written planar Bragg grating sensors. Meas. Sci. Technol. 2015, 26, 112001. [CrossRef]

15. Bhatta, D.; Michel, A.A.; Marti Villalba, M.; Emmerson, G.D.; Sparrow, I.J.G.; Perkins, E.A.; McDonnell, M.B.; Ely, R.W.; Cartwright, G.A. Optical microchip array biosensor for multiplexed detection of bio-hazardous agents. Biosens. Bioelectron. 2011, 30, 78-86. [CrossRef] [PubMed]

16. Wales, D.J.; Parker, R.M.; Quainoo, P.; Cooper, P.A.; Gates, J.C.; Grossel, M.C.; Smith, P.G.R. An integrated optical Bragg grating refractometer for volatile organic compound detection. Sens. Actuators B Chem. 2016, 232, 595-604. [CrossRef]

17. Girschikofsky, M.; Rosenberger, M.; Belle, S.; Brutschy, M.; Waldvogel, S.R.; Hellmann, R. Highly sensitive detection of naphthalene in solvent vapor using a functionalized PBG refractive index sensor. Sensors 2012, 12, 2018-2025. [CrossRef]

18. Girschikofsky, M.; Ryvlin, D.; Waldvogel, S.R.; Hellmann, R. Optical Sensor for Real-Time Detection of Trichlorofluoromethane. Sensors 2019, 19, 632. [CrossRef]

19. Eldada, L.; Shacklette, L.W. Advances in polymer integrated optics. IEEE J. Select. Top. Quantum Electron. 2000, 6, 54-68. [CrossRef]

20. Ziemann, O.; Daum, W.; Krauser, J.; Zamzow, P.E. POF Handbook: Optical Short Range Transmission Systems, 2nd ed.; Springer: Berlin/Heidelberg, Germany, 2008.

21. Rosenberger, M.; Eisenbeil, W.; Schmauss, B.; Hellmann, R. Simultaneous 2D strain sensing using polymer planar Bragg gratings. Sensors 2015, 15, 4264-4272. [CrossRef] [PubMed]

22. Rosenberger, M.; Pauer, H.; Girschikofsky, M.; Woern, H.; Schmauss, B.; Hellmann, R. Flexible Polymer Shape Sensor Based on Planar Waveguide Bragg Gratings. IEEE Photonics Technol. Lett. 2016, 28, 1898-1901. [CrossRef]

23. Micro Resist Technology GmbH. EpoCore/EpoClad Datasheet. Available online: http://www.microresist.de (accessed on 16 May 2021).

24. Prajzler, V.; Neruda, M.; Nekvindova, P.; Mikulik, P. Properties of Multimode Optical Epoxy Polymer Waveguides Deposited on Silicon and TOPAS Substrate. Radioengineering 2017, 26, 10-15. [CrossRef]

25. Missinne, J.; Teigell Benéitez, N.; Lamberti, A.; Chiesura, G.; Luyckx, G.; Mattelin, M.-A.; van Paepegem, W.; van Steenberge, G. Thin and Flexible Polymer Photonic Sensor Foils for Monitoring Composite Structures. Adv. Eng. Mater. 2018, $20,1701127$. [CrossRef]

26. Hessler, S.; Bott, P.; Kefer, S.; Schmauss, B.; Hellmann, R. Multipurpose Polymer Bragg Grating-Based Optomechanical Sensor Pad. Sensors 2019, 19, 4101. [CrossRef]

27. Meyer, J.; Nedjalkov, A.; Kelb, C.; Strobel, G.J.; Ganzer, L.; Schade, W. Manufacturing and Characterization of Femtosecond Laser-Inscribed Bragg Grating in Polymer Waveguide Operation in an IR-A Wavelength Range. Sensors 2020, 20, 249. [CrossRef] [PubMed]

28. Hessler, S.; Schmauss, B.; Hellmann, R. Temperature corrected lab-on-a-chip-platform with integrated epoxy polymer Bragg gratings. In Proceedings of the Optical Sensing and Detection V, Strasbourg, France, 9 May 2018; pp. 27-36. [CrossRef]

29. Hessler, S.; Rüth, M.; Sauvant, C.; Lemke, H.-D.; Schmauss, B.; Hellmann, R. Hemocompatibility of EpoCore/EpoClad photoresists on COC substrate for optofluidic integrated Bragg sensors. Sens. Actuators B Chem. 2017, 239, 916-922. [CrossRef]

30. Hessler, S.; Knopf, S.; Rommel, M.; Girschikofsky, M.; Schmauss, B.; Hellmann, R. Advancing the sensitivity of integrated epoxy-based Bragg grating refractometry by high-index nanolayers. Opt. Lett. 2020, 45, 5510-5513. [CrossRef] [PubMed]

31. Kashyap, R. Fiber Bragg Gratings, 2nd ed.; Academic Press: Burlington, NJ, USA, 2010.

32. Othonos, A.; Kalli, K. Fiber Bragg Gratings. Fundamentals and Applications in Telecommunications and Sensing; Artech House: Norwood, MA, USA, 1999.

33. Broadway, C.; Min, R.; Leal-Junior, A.G.; Marques, C.; Caucheteur, C. Toward Commercial Polymer Fiber Bragg Grating Sensors: Review and Applications. J. Lightwave Technol. 2019, 37, 2605-2615. [CrossRef]

34. Hoppe, J.-D.; Scriba, P.C.; Klüter, H. GmbH Freiburg. Human Albumin. Transfus. Med. Hemotherapy 2009, 36, 399-407. [CrossRef]

35. R Diaz, C.A.; Leal-Junior, A.G.; M Avellar, L.; C Antunes, P.F.; Pontes, M.J.; Marques, C.A.; Frizera, A.; N Ribeiro, M.R. Perrogator: A Portable Energy-Efficient Interrogator for Dynamic Monitoring of Wavelength-Based Sensors in Wearable Applications. Sensors 2019, 19, 2962. [CrossRef] [PubMed] 